\title{
Evaluación de la calidad del agua en los ríos Tigre y Rincón de la península de Osa en dos períodos de tiempo distintos
}

\author{
Water quality evaluation in Tigre and Rincón rivers \\ of península de Osa in two different periods of time
}

\section{Guillermo Calvo-Brenes ${ }^{1}$, Jesús Mora-Molina²}

Fecha de recepción: 12 de diciembre del 2014 Fecha de aprobación: 18 de marzo del 2015

Calvo-Brenes, G; Mora-Molina, J. Evaluación de la calidad del agua en los ríos Tigre y Rincón de la península de Osa en dos períodos de tiempo distintos. Tecnología en Marcha.

Vol. 28, Nº 3, Julio-Setiembre. Pág 55-63. 


\section{Palabras clave}

Contaminación de ríos; coliformes fecales; índice de calidad de agua; Índice Holandés; río Rincón; río Tigre; península de Osa; Costa Rica.

\section{Resumen}

La península de Osa es una zona que se caracteriza por una alta biodiversidad, tanto en su flora como en su fauna. El recurso hídrico no solo es un indicador de la condición económica y social de una región, sino que está estrechamente ligada con la protección de su flora y su fauna. Costa Rica es un país privilegiado por la cantidad de ríos y mantos acuíferos que posee; sin embargo, el incremento en los niveles de contaminación que estos han experimentado es preocupante y la península de Osa no escapa a esta realidad nacional.

El objetivo del estudio fue evaluar la calidad en dos ríos importantes de la península: el Tigre y el Rincón, en dos períodos de tiempo diferentes para verificar si la calidad del agua ha cambiado a lo largo del tiempo evaluado. Los niveles de contaminación se determinaron por medio del Índice Holandés y también por su contenido en coliformes fecales. En cada uno de los ríos se evaluó la parte alta y la baja ya que las condiciones socioambientales son diferentes. Los periodos evaluados fueron el 2008-2009 y el 2010-2011. Además, los datos recolectados se segregaron en dos épocas: la lluviosa y la seca, con el fin de facilitar el análisis estadístico empleando el programa de cómputo estadístico SPSS.

Se encontró que el contenido de coliformes fecales durante la época seca es tal que permite el uso de esos ríos para actividades recreativas de contacto primario en ambos períodos de tiempo evaluados. En cambio, en la época de invierno, el contenido de coliformes fecales superó los niveles de permisibilidad para poder efectuar actividades recreativas en el año 2009. En el caso del invierno del 2011, los niveles de coliformes fecales estuvieron por debajo del nivel máximo de permisibilidad para tal uso, excepto para el río Tigre, en su zona alta en el mes de julio, y para el mismo río en su zona baja en el de Junio, que tuvieron valores no aptos para practicar la natación.

El nivel de contaminación de los puntos muestreados, según el Índice Holandés, oscila entre niveles de No Contaminación hasta Contaminación Leve. El análisis estadístico no mostró diferencias significativas entre la época de invierno y la de verano, o entre los períodos de tiempo evaluados del 2008-2009 y el 2010-2011, dada la variabilidad de los datos entre épocas por punto de muestreo en un período dado. La única excepción es el punto del río Tigre en su zona baja (Ti-3) que sí presenta un incremento estadístico en los niveles de contaminación, entre un período y otro, durante la época de invierno.

\section{Keywords}

River contamination; Fecal Coliforms; water quality index; Dutch Index; Rincón river; Tigre river; península of Osa; Costa Rica.

\section{Abstract}

Península of Osa is a region characterized by a high biodiversity in plants and animals, as well. Hydric resources are not only an indicator of economic and social condition of a region but also related to protection of plants and animals. Costa Rica is privileged by the many rivers and ground waters it has; but the contamination levels are increasing alongside and península of Osa does not scape to that country reality. 
The objective of this research was to evaluate the quality of two important rivers of the peninsula: Tigre and Rincón rivers in two different periods of time in order to verify if rivers quality has changed during the time evaluated. Contamination levels were determined by the Dutch Index and Fecal Coliforms content in waters. On each river the evaluation was done in the upper side and the lower side since social and environmental conditions are different. The periods of time selected for evaluation were 2008-2009 and 2010-2011. Besides, data collected was separated in two seasons: summer and winter, so that statistical analysis can be performed using the statistical software SPSS.

It was found that Fecal Coliforms content during summer time is such that recreational activity of first contact is allowed in those two periods of time. On the other hand, the Fecal Coliforms content during winter of 2009 exceeded the limits so recreational activity is not allowed. In general, the Fecal Coliforms content in 2011 were below the limits, except for the upper side area in July for Tigre river(Ti-1) and the lower side area in June for Tiger river (Ti-3).

The contamination levels of each sampled zones ranges the No Contamination level and Little Contamination level when evaluated through the Dutch Index. Statistical analysis does not show any statistical difference between summer and winter or between time periods (2008-2009 and 2010-2011). The only exception is the lower side of Tigre river (Ti-3) that shows a statistical increase in the contamination level between the two time periods evaluated during winter.

\section{Introducción}

la península de Osa está localizada en la costa suroeste del océano Pacífico y se caracteriza por poseer una biodiversidad alta, raramente encontrada en un lugar pequeño. Aproximadamente un tercio de las especies de árboles existentes en Costa Rica se han registrado en la región incluyendo la mitad de las especies de árboles amenazadas en el país. Se estima que existen entre 4000 y 5000 especies de plantas vasculares en la península. La variedad de fauna es extraordinariamente rica: se han registrado unas 375 especies de aves, 124 especies de mamíferos, 40 especies de peces de agua dulce y, aproximadamente, 8000 especies de insectos. Las especies registradas en la región representan entre el 30\%-50\% de todas las especies conocidas en el país (Rosero, Maldonado y Bonilla, 2007). Algunos expertos identifican en la península tres zonas de vida y cuatro transiciones: Bosque Húmedo Tropical, Bosque Húmedo Tropical transición a Prehúmedo, Bosque muy Húmedo Tropical, Bosque muy Húmedo Tropical transición a Premontano, Bosque muy Húmedo Premontano transición a Basal, Bosque Pluvial Premontano y Bosque Pluvial Premontano transición a Basal (Fundación Neotrópica, 1992). Basado en los atributos ecológicos claves como objetos de conservación de la región se distinguen las siguientes categorías: Bosque Basal Lluvioso del Pacífico, Bosque Nuboso, Bosques Anegados, Manglares, Ecosistemas Lóticos, Ecosistemas Lénticos y Felinos Grandes (Ugalde et al, 2007).

El uso del agua es el mejor indicador del grado de desarrollo social y económico de un país. El agua es un elemento esencial no solo para la preservación de la vida sino, también, para la conservación de la flora y fauna en una región, lo que representa, en algunos casos, el atractivo turístico de la zona (Sánchez, 2009). La Organización Mundial de la Salud ha dicho que el 80\% de las enfermedades del mundo se debe a problemas con el agua (AyA, 2003; OMCO, 2010; FAO, 2011). La tifoidea, el cólera, la disentería, la diarrea y la hepatitis infecciosa son cinco enfermedades que se transmiten por agua contaminada o por lavar alimentos, utensilios y manos en ella (OMS, 2009). La malaria y la fiebre amarilla son enfermedades transmitidas por vectores que se crían en el agua (OMS, 2011). 
Costa Rica es un país privilegiado por la cantidad de ríos y mantos acuíferos que posee. Desafortunadamente, los niveles de contaminación de los ríos que atraviesan zonas de alta densidad poblacional, son altos (Calvo y Mora, 2007).

El objetivo de la investigación es evaluar el cambio en la calidad del agua que se han dado en dos períodos distintos: 2008-2009 y 2010-2011. El análisis de la calidad del agua se llevó a cabo por medio del Índice Holandés de evaluación de la calidad del agua, el cual clasifica la calidad del agua en 5 clases, de acuerdo a la legislación costarricense (MINAE, 2007). También se evaluó el contenido de coliformes fecales (CF), un indicador muy importante de nuestra legislación que establece como límite contenidos máximos de 1000 CF para que el agua se pueda usar con fines recreativos de contacto primario, como lo es la práctica de la natación.

\section{Metodología}

el estudio se llevó a cabo en dos subcuencas que forman parte de la península de Osa: la del río Tigre y la del río Rincón (figura 1). En cada subcuenca se muestreó el río principal en su zona alta, lo más cerca posible de la naciente del río; así como en su zona baja, cerca de la desembocadura. Las zonas altas y bajas en ese sector del estudio tienen características geomorfológicas y socioambientales diferentes. La zona alta en cada una de estas subcuencas se caracteriza por pendientes altas, densidad poblacional nula y alta cobertura vegetal que forma parte del Parque Nacional Corcovado. En cambio, las zonas bajas de caracterizan por ser de pendiente muy baja, densidad poblacional baja y uso del suelo con fines agrícolas, aunque también existe cobertura boscosa en esta zona baja.

Las muestras recolectadas en el río Rincón se codificaron como R1 (zona alta) y R3 (zona baja); mientras que el río Tigre se clasificaron como Ti 1 (zona alta) y Ti3 (zona baja).

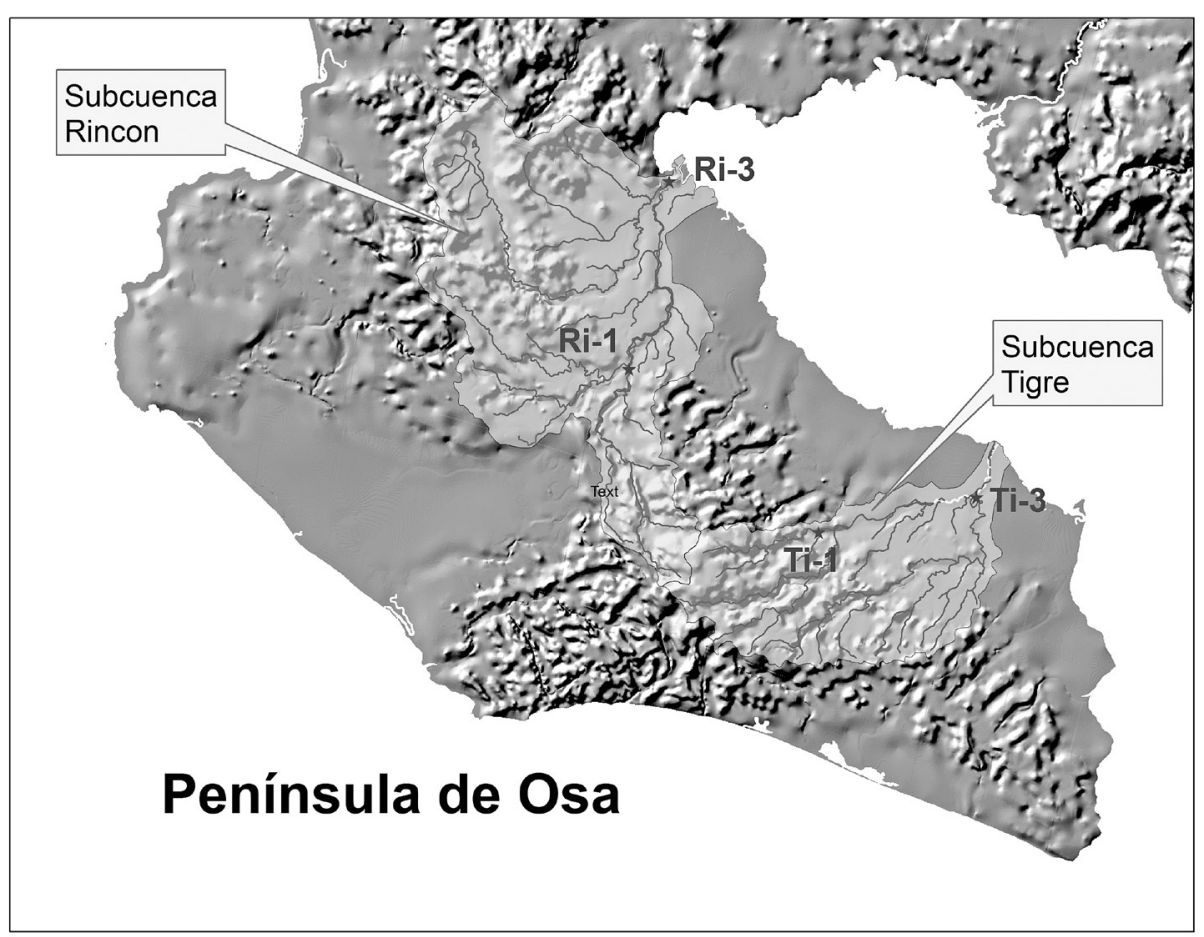

Figura 1. Subcuencas seleccionadas pertenecientes a la península de Osa. Fuente: Mapa elaborado por Guillermo Calvo, 2014. 
La localización geográfica de los puntos de muestreo se indica en el cuadro 1.

Cuadro 1. Georreferenciación y características de los puntos de muestreo en la península de Osa

\begin{tabular}{|c|c|c|c|c|c|}
\hline \multirow{2}{*}{$\begin{array}{l}\text { Puntos de } \\
\text { muestreo }\end{array}$} & \multicolumn{2}{|c|}{ Coordenadas geográficas } & \multirow{2}{*}{$\begin{array}{l}\text { Altitud } \\
\text { (m) }\end{array}$} & \multirow{2}{*}{$\begin{array}{c}\text { Densidad } \\
\text { poblacional } \\
\left(\mathrm{hab} / \mathrm{km}^{2}\right)\end{array}$} & \multirow{2}{*}{ Características } \\
\hline & Latitud norte & Longitud oeste & & & \\
\hline $\mathrm{Ri}-1$ & $8^{\circ} 36^{\prime} 11.78 ” \mathrm{~N}$ & $83^{\circ} 29^{\prime} 48.84^{\prime \prime} \mathrm{W}$ & 37 & 0 & $\begin{array}{c}\text { Baja densidad poblacional. } \\
\text { Cobertura boscosa alta. }\end{array}$ \\
\hline $\mathrm{Ri}-3$ & $8^{\circ} 41^{\prime} 23.41^{\prime \prime} \mathrm{N}$ & $83^{\circ} 28^{\prime} 40.95^{\prime \prime} \mathrm{W}$ & 24 & 2 & $\begin{array}{l}\text { Cobertura boscosa media } \\
\text { Actividad agrícola alta }\end{array}$ \\
\hline $\mathrm{Ti}-1$ & $8^{\circ} 31 ' 39.77^{\prime \prime} \mathrm{N}$ & $83^{\circ} 24^{\prime} 32.97^{\prime \prime} \mathrm{W}$ & 108 & 4 & $\begin{array}{l}\text { Baja densidad poblacional. } \\
\text { Cobertura boscosa alta. }\end{array}$ \\
\hline Ti-3 & $8^{\circ} 32^{\prime} 37.45^{\prime \prime} \mathrm{N}$ & $83^{\circ} 20^{\prime} 8.77^{\prime \prime} \mathrm{W}$ & 0 & 0 & $\begin{array}{c}\text { Cobertura boscosa media } \\
\text { Actividad agrícola alta }\end{array}$ \\
\hline
\end{tabular}

La evaluación de la calidad de las aguas se llevó a cabo en dos períodos de tiempo: 2008-2009 y 2010-2011. En el caso del análisis de CF, el período de evaluación fue de enero hasta agosto, en el año 2009 y 2011, respectivamente. La cuantificación de este indicador se efectuó por el método de fermentación de tubos múltiples, conocidos como número más probable (NMP/100 $\mathrm{ML}$ ), indicado en el Standard Methods for the Examination of Water and Wastewater" (APHA, 2005).

La clasificación de la calidad de las aguas en 5 clases diferentes se llevó a cabo utilizando el Índice Holandés para valorar la calidad en cuerpos de agua superficiales. El Índice se basa en la obtención de un puntaje de acuerdo con tres indicadores analizados: la demanda bioquímica de oxígeno $\left(\mathrm{DBO}_{5}\right)$, el oxígeno disuelto $(\mathrm{OD})$ y el nitrógeno amoniacal $\left(\mathrm{N}-\mathrm{NH}_{4}{ }^{+}\right)$. El $\mathrm{OD}$ es un indicador de calidad medido en el campo y con este indicador se puede calcular el porcentaje de saturación de oxígeno (PSO), indicador necesario para la clasificación de la calidad del agua. El cuadro 2 muestra el puntaje dado a cada indicador, según su concentración en el agua y la sumatoria de estos tres datos se compara con la información dada en el cuadro 3 para clasificar la calidad del agua (MINAE, 2007).

Cuadro 2. Asignación de puntaje para $\mathrm{PSO}, \mathrm{DBO}_{5}$ y del $\mathrm{N}-\mathrm{NH}_{4}{ }^{+}$, según el Índice Holandés de Valoración de la calidad del agua

\begin{tabular}{|c|c|c|c|}
\hline PUNTOS & PSO, \% & $\mathrm{DBO}, \mathrm{mg} / \mathrm{L}$ & $\mathrm{N}-\mathrm{NH}^{+4}, \mathrm{mg} / \mathrm{L}$ \\
\hline 1 & $91-100$ & $<=3$ & $<0.50$ \\
\hline 2 & $\begin{array}{c}71-90 \\
111-120\end{array}$ & $3.1-6.0$ & $0.50-1.0$ \\
\hline 3 & $\begin{array}{c}51-70 \\
121-130\end{array}$ & $6.1-9.0$ & $1.1-2.0$ \\
\hline 4 & $31-50$ & $9.1-15$ & $2.1-5.0$ \\
\hline 5 & $<=30 y>130$ & $>15$ & $>5.0$ \\
\hline
\end{tabular}

Fuente: MINAE, 2007 
La metodología, tanto para el análisis como para la toma de las muestras de los indicadores del Índice Holandés, fue la establecida en el "Standard MethodsfortheExamination of Water and Wastewater (APHA, 2005).

Cuadro 3. Clasificación numérica de las variables según la metodología del Índice Holandés de Valoración

\begin{tabular}{|c|c|c|c|}
\hline Clase & $\begin{array}{c}\text { Promedio de } \\
\text { puntos }\end{array}$ & Código de color & $\begin{array}{c}\text { Interpretación de } \\
\text { calidad }\end{array}$ \\
\hline 1 & 3 & Azul & Sin contaminación \\
\hline 2 & $4-6$ & Verde & $\begin{array}{c}\text { Contaminación } \\
\text { incipiento }\end{array}$ \\
\hline 3 & $7-9$ & Amarillo & $\begin{array}{c}\text { Contaminación } \\
\text { moderada }\end{array}$ \\
\hline 4 & $10-12$ & Anaranjado & $\begin{array}{c}\text { Contaminación } \\
\text { severa }\end{array}$ \\
\hline 5 & $13-15$ & Rojo & $\begin{array}{c}\text { Contaminación } \\
\text { muy severa }\end{array}$ \\
\hline
\end{tabular}

Fuente: MINAE, 2007

La verificación de posibles cambios en los niveles de contaminación del agua en cada uno de los puntos de muestreo en ambos períodos de tiempo se llevó a cabo utilizando el programa de cómputo estadístico Statistical Package for Social Science (SPSS). Los distintos valores de contaminación medidos por medio del Índice Holandés, durante 12 meses en cada uno de los puntos de muestreo, se segregaron en dos épocas diferentes: la lluviosa y la seca. El Instituto Meteorológico Nacional estableció que la época seca correspondió desde mediados del mes de noviembre hasta abril del siguiente año, y la lluviosa desde el mes de mayo hasta mediados de noviembre del mismo año. Por conveniencia para dicho análisis, se redefinió la época seca de los meses de noviembre a abril y la lluviosa desde mayo hasta octubre. Las posibles diferencias entre puntos de muestreo por época y por período de tiempo se evaluaron por medio del Análisis de Varianza (ANOVA) y empleando la metodología de Duncan con un nivel de significancia de un 5\%, para poder determinar posibles diferencias entre grupos distintos.

\section{Discusión de los resultados}

El cuadro 4 muestra los resultados del análisis de CF entre los años 2009 y 2011 en cada uno de los puntos de muestreo. Se observa que el contenido de CF en el 2011 fue menor, en comparación con el período 2009. En dicho cuadro se clasificaron los contenidos de CF menores a $1000 \mathrm{NMP} / 100 \mathrm{ml}$ con el color verde, indicando con ello que se pueden utilizar con fines recreativos de contacto primario, de acuerdo con los límites de permisibilidad establecido en nuestro reglamento. Por otra parte, aquellos datos en color rojo corresponden a valores de CF superiores a los $1000 \mathrm{NMP} / 100 \mathrm{ml}$ cuyo uso no puede dedicarse a actividades como la natación.

Los meses con color rojo fueron más frecuentes en el 2009,reduciendo el tiempo de uso recreativo durante el año en ambos ríos; mientras que en el período 2011, solo estuvo restringido un mes para tal actividad recreativa; y únicamente en el río Tigre el mes de julio para la zona baja, y el mes de junio en la zona alta. 
El cuadro 4 muestra que los niveles de contaminación por CF se incrementaron en el período de Iluvia, mientras que la contaminación por CF es menor en la época seca. En general, se considera que el incremento en CF está asociado con el incremento en los períodos de lluvia; sin embargo, no fue factible corroborarlo debido a la ausencia de estaciones pluviales localizadas en las zonas de muestreo, La península de Osa se caracteriza por poseer microclimas distintos ubicados en distintas zonas de la región, y tanto la subcuenca del río Tigre como la del río Rincón se caracterizan por comportamientos pluviales bastante diferentes entre sí.

Cuadro 4. Comparación de contaminación fecal encontrada en las muestras de aguas recolectadas en el año 2009 y año 2011.

\begin{tabular}{|c|c|c|c|c|c|c|c|c|c|}
\hline \multicolumn{8}{|c|}{ Cantidad de coliformes fecales (NMP/100 ML) año 2009 } \\
\hline Ríos & Ene & Feb & Mar & Abr & May & Jun & Jul & Ago \\
\hline Ri-1 & 240 & 75 & 240 & 1100 & 2100 & 4600 & 460 & 1100 \\
\hline Ri-3 & 240 & 43 & 150 & 460 & 1100 & 4600 & 460 & 46000 \\
\hline Ti-1 & 43 & 43 & 75 & 150 & 93 & 1500 & 460 & 1100 \\
\hline Ti-3 & 93 & 460 & 93 & 240 & 2400 & 4600 & 460 & 1100 \\
\hline \multicolumn{7}{|c|}{ Cantidad de coliformes fecales (NMP/100 ML) año 2011 } \\
\hline Ríos & Ene & Feb & Mar & Abr & May & Jun & Jul & Ago \\
\hline Ri-1 & 9 & 43 & 43 & 23 & 23 & 43 & 43 & 9 \\
\hline Ri-3 & 43 & 23 & 9 & 93 & 460 & 460 & 460 & 460 \\
\hline Ti-1 & 23 & 9 & 43 & 4 & 23 & 43 & 11000 & 43 \\
\hline Ti-3 & 23 & 4 & 15 & 460 & 460 & 11000 & 460 & 460 \\
\hline
\end{tabular}

El cuadro 5 muestra los resultados encontrados en los cuatro puntos de muestreo seleccionados y distribuidos en las dos subcuencas en el período 2008-2009 y 2010-2011, que corresponden a la aplicación del Índice Holandés. En el período 2010-2011 el orden de los meses se reacomodó en el Cuadro 5 para que el proceso comparativo por mes sea visualmente más fácil en su interpretación.

Cuadro 5. Comparación de la calidad del agua según el Sistema Holandés en las muestras de aguas recolectadas en el año 2008-2009 y año 2010-2011.

\begin{tabular}{|c|c|c|c|c|c|c|c|c|c|c|c|c|}
\hline \multicolumn{10}{|c|}{ Muestreos realizados entre setiembre del 2008 hasta agosto del 2009.} \\
\hline Ríos & Set & Oct & Nov & Dic & Ene & Feb & Mar & Abr & May & Jun & Jul & Ago \\
\hline Ri-1 & 4 & 4 & 3 & 5 & 5 & 3 & 5 & 3 & 4 & 4 & 5 & 4 \\
\hline Ri-3 & 4 & 4 & 4 & 4 & 4 & 3 & 4 & 4 & 4 & 4 & 5 & 4 \\
\hline Ti-1 & 4 & 4 & 3 & 3 & 4 & 3 & 3 & 3 & 3 & 4 & 3 & 6 \\
\hline Ti-3 & 4 & 4 & 3 & 3 & 4 & 3 & 3 & 3 & 3 & 4 & 4 & 3 \\
\hline \multicolumn{7}{|c|}{} & Muestreos realizados entre noviembre del 2010 hasta octubre del 2011. & \\
\hline Ríos & Set & Oct & Nov & Dic & Ene & Feb & Mar & Abr & May & Jun & Jul & Ago \\
\hline Ri-1 & 4 & 5 & 3 & 4 & 5 & 3 & 3 & 3 & 4 & 5 & 9 & 4 \\
\hline Ri-3 & 4 & 5 & 4 & 5 & 4 & 3 & 3 & 4 & 3 & 5 & 6 & 4 \\
\hline Ti-1 & 4 & 5 & 3 & 4 & 5 & 4 & 3 & 4 & 3 & 5 & 11 & 4 \\
\hline Ti-3 & 4 & 5 & 3 & 5 & 4 & 3 & 3 & 4 & 7 & 5 & 10 & 5 \\
\hline
\end{tabular}


Primeramente se observa que los niveles de contaminación en cada uno de los puntos muestreados oscila entre la categoría de No Contaminado (azul) a Levemente Contaminado (verde), excepto en el mes de julio del 2011 donde, claramente, hay un incremento en los niveles de contaminación. Los mismos pudieron estar asociados a un incremento en los niveles de lluvia que fueran particularmente altos. Sin embargo, la falta de datos de precipitación no permite el probar tal hipótesis.

Por otra parte, los datos individuales en cada uno de los puntos de muestreo debieron agruparse por época seca y lluviosa para llevar a cabo el análisis estadístico. El análisis de cambios en niveles de contaminación, al comparar cada punto de muestreo dentro de una misma época pero de diferente período 2008-2009 en relación con el 2010-2011, mostró que no existe diferencia estadística significativa para justificar alguna variabilidad, excepto para el punto Tigre-3 donde sí hubo un incremento en los niveles de contaminación

\section{Conclusiones}

Los niveles de CF en la época seca en ambos períodos evaluados presentaron condiciones adecuadas para el uso del agua con fines recreativos en cada uno de los puntos de muestreo. La época lluviosa mostró condiciones diferentes entre ambos períodos de estudio. En el 2011 hubo condiciones para el uso recreativo del agua en todos los puntos de muestreo, excepto para Ti-1 en Julio y Ti-3 en Junio. En cambio, la época lluviosa del año 2009 presentó prácticamente condiciones de contaminación no aptas para el uso del agua para actividades recreativas.

El análisis estadístico del nivel de contaminación evaluado con el Índice Holandés en cada uno de los puntos de muestreo no indica cambios significativos entre períodos ni entre época del año. La única excepción es el punto Ti-3 que sí muestra un cambio significativo en los niveles de contaminación durante la época lluviosa, el cual es mayor en el año 2011.

\section{Agradecimientos}

Los autores expresan sus agradecimientos al Consejo Nacional de Rectores (CONARE), por el apoyo financiero, y al Instituto Tecnológico de Costa Rica (ITCR), en especial a la Vicerrectoría de Investigación y Extensión (VIE). También, agradecemos al Centro de Investigación en Protección Ambiental (CIPA) y al Laboratorio de Servicios Químicos y Microbiológicos (CEQIATEC) por su gran respaldo al proyecto.

\section{Bibliografía}

APHA. (2000). Standard methods for the examination of water and wastewater, $20{ }^{\circ}$ edición, Digital.

AyA. 2003. VII Congreso Nacional de ingeniería sanitaria y ambiental. Simposio realizado en el Colegio Federado de Ingenieros y Arquitectos, San José, Costa Rica.

Calvo, G. y Mora, J. 2007. Evaluación y clasificación preliminar de la calidad de agua de la cuenca del Río Tárcoles y el Reventazón. Parte III.: Calidad de cuerpos receptores de agua, según el Sistema Holandés de Valoración, 20(4), 59-67.

FAO. 2011. La FAO lanza campaña para prevenir las enfermedades de transmisión alimentaria. Recuperado el 11 de noviembre, 2011 desde http://www.alimentacion.enfasis.com/notas/12574-la-fao-lanza-campana-prevenir-lasenfermedades-trasmision-alimentaria.

Fundación Neotrópica. (1992). Evaluación ecológica rápida península de Osa. Programa BOSCOSA de la Fundación Neotrópica. Informe Final, agosto de 1992. 
MINAE. 2007. Reglamento para la Clasificación y Evaluación de la Calidad de Cuerpos de Agua Superficiales para la clasificación y la evaluación de la calidad de cuerpos de agua superficiales. No 33903 MINAE-S. Gaceta \#178, 17 de setiembre del 2007. San José, Costa Rica.

OMCO. 2010. Estadísticas de enfermedades provocadas por el agua.Consumer World Organization. Recuperado el 13 de febrero, 2010 desde .

OMS, 2009. Enfermedades diarreicas, una amenaza latente. Recuperado el 13 de febrero, 2010 desde http://elmercuriodigital.es-elmercuriodigital.es.

OMS, 2011. Agua, saneamiento y salud: Manejo ambiental para el control de vectores. Recuperado el 11 de noviembre, 2011 desde http://www.who.int/water_sanitation_health/resources/envmanagement/es/index.html

Rosero, L; Maldonado, T. y Bonilla, R. 2007. Bosque y población en la península de Osa. Recuperado el 1 de diciembre, 2014 desde http://ccp.ucr.ac.cr/seminario/pdf/rosero.pdf

Sanchez V., A. 2009. Antología curso: geodinámica y problemas ambientales Doctorado en Ciencias Naturales para el desarrollo, 3. ${ }^{\circ}$ promoción 2009, Universidad de Chapingo, México.

Ugalde, J. et al. 2007. Programa de monitoreo ecológico del estado de conservación del sitio Osa. Documento de trabajo. INBIO. 